\title{
Cryptogenic Cirrhosis
}

National Cancer Institute

\section{Source}

National Cancer Institute. Cryptogenic Cirrhosis. NCI Thesaurus. Code C84411.

Cirrhosis in which no causative agent can be identified. 